\title{
Comparison and Evaluation of Anti-Windup PI Controllers
}

\author{
Xin-lan Li*, Jong-Gyu Park**, and Hwi-Beom Shin ${ }^{\dagger}$ \\ $\dagger^{*}$ Dept. of Electrical Engineering, Gyeongsang National University, Gyeongnam, Korea \\ ${ }^{* *}$ Namhae College, Gyeongnam, Korea
}

\begin{abstract}
This paper proposes a method for comparing and evaluating anti-windup proportional-integral (PI) control strategies. The so-called PI plane is used and its coordinate is composed of the error and the integral state. In addition, an anti-windup PI controller with integral state prediction is proposed. The anti-windup scheme can be easily analyzed and evaluated on the PI plane in detail. Representative anti-windup methods are experimentally applied to the speed control of a vector-controlled induction motor driven by a pulse width modulated (PWM) voltage-source inverter (VSI). The experimental results compare the anti-windup PI controllers. It is empathized that the initial value of the integral state at the beginning of the linear range dominates the control performance in terms of overshoot and settling time.
\end{abstract}

Key Words: Anti-windup, Integral state prediction, PI controller, Variable-speed motor drives

\section{INTRODUCTION}

PI control has been widely used as a cascaded form of control in variable-speed motor drives. The current control is employed in an inner feedback loop for fast dynamics and peak current protection and the outer speed controller generates a current command for the current controller. Since the current command is typically limited to a prescribed maximum value, the integral state is not consistent with the plant input and becomes very large, which causes the integral windup phenomenon. There exists a large overshoot, a slow settling time, and, sometimes, even instability in the speed response [1], [2].

A number of the anti-windup schemes have been proposed in the literature and may be representatively classified into three categories which include the conditional integration, the tracking back calculation, and the limited integrator schemes. For the conditional integration schemes [3]-[6], the integral action is suspended when the control input is saturated and the $\mathrm{P}$ control only is activated. The PI control is effective when the control input lies within the saturation limit. The difference between the saturated and the unsaturated control input signals is used to generate a feedback signal to act on the integrator input for the tracking back calculation methods [1], [7]-[9]. The transient performance such as the overshoot depends heavily upon the feedback gain of the control difference rather than the PI gains. For the limited integrator scheme [10], the

\footnotetext{
Manuscript received Aug. 13, 2010; revised Nov. 4, 2010

$\dagger$ Corresponding Author: hbshin@gnu.ac.kr

Tel: +82-55-751-5348, Fax: +82-55-759-2723, Gyeongsang Univ.

* Dept. of Electrical Eng., Gyeongsang Nat'l University, Korea

** Namhae College, Korea
}

integrator value is limited by feeding the control back with a high-gain dead zone in order to guarantee operation in the linear range. In case of a saturation-type limit, this scheme is same as the back calculation method.

It is very difficult and complex to compare and evaluate anti-windup PI controllers since we should consider the overall operating conditions such as the possible load and command changes as well as the manual/auto transfer operations, etc.

In this paper, a method is proposed for comparing and evaluating anti-windup PI control schemes. An anti-windup PI controller with the integral state prediction is also proposed. The PI plane is utilized and its coordinate is composed of the error and the integral state. The anti-windup scheme can be easily analyzed and evaluated in detail on the PI plane. Representative anti-windup methods are experimentally applied to the speed control of a vector-controlled induction motor driven by a pulse width modulated (PWM) voltagesource inverter (VSI). Through the experimental results, the anti-windup PI controllers are compared and evaluated.

\section{PI PLANE AND THE LINEAR RANGE OF PI CONTROL}

It is common that an inner current controller is designed to have much faster dynamics than an outer speed controller in a cascaded form. If a fast current control scheme is employed, the current dynamics is negligible and a variable-speed motor drive can be considered as a first-order system given by:

$$
\frac{d \omega_{r}}{d t}=-\frac{1}{\tau_{m}} \omega_{r}+k_{t} v-T_{l}
$$

where $\tau_{m}=J / B, k_{t}=k_{T} / J, T_{l}=T_{L} / J$ and $v$ denotes the plant input, namely, the torque-producing current [11]. It is 


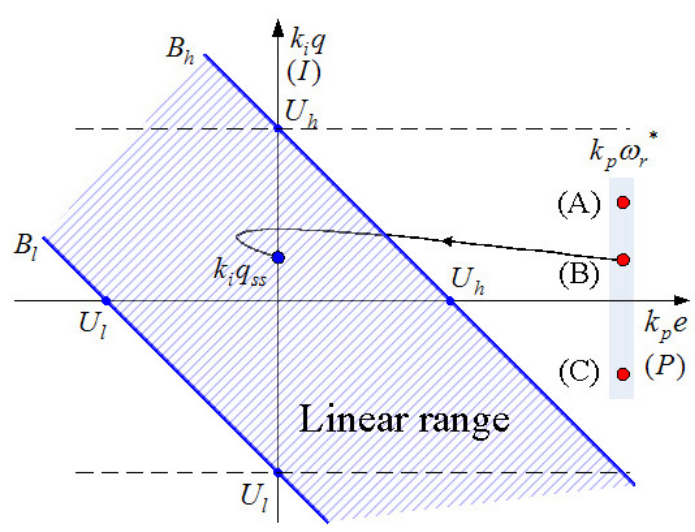

Fig. 1. Linear range of PI control in PI plane.



Fig. 2. Error trajectories according to touch conditions.

assumed that the plant input $v$ is limited by a saturation-type nonlinearity as:

$$
v=\left\{\begin{array}{ccc}
U_{h} & \text { if } & u>U_{h} \\
u & \text { if } & U_{l} \leq u \leq U_{h} \\
U_{l} & \text { if } & u<U_{l}
\end{array}\right.
$$

where $u$ represents the controller output. In the following, this will be called a linear range and a saturation range when $u=v$ and $u \neq v$, respectively.

The PI control law is expressed by:

$$
u=k_{p} e+k_{i} q
$$

where $k_{p}$ and $k_{i}$ denote the proportional and integral gains, respectively. Their error is:

$$
e=\omega_{r}^{*}-\omega_{r}
$$

where $\omega_{r}^{*}$ denotes the speed reference. The integral state $q$ is dependant upon the anti-windup scheme.

If the plant input constraint in (2) is considered, the upper and lower boundaries of the linear range can be found from (3) as:

$$
\begin{array}{ll}
B_{h}: & I=-P+U_{h} \\
B_{l}: & I=-P+U_{l}
\end{array}
$$

where $I$ and $P$ denote the integral and proportional actions, respectively, and $I=k_{i} q$ and $P=k_{p} e$. Fig. 1 shows the linear range in $k_{p} e-k_{i} q$ or the $P-I$ axis, which will be called the PI plane in the following. If the operating point $\left(k_{p} e, k_{i} q\right)$ lies outside the linear range, the control input is saturated and the integral state is not consistent with the plant input. The $\mathrm{P}$ action only is effectively activated. From Fig. 1, the steady state value of the integral state $k_{i} q_{s s}$ can be written, if the PI control system is stable, as:

$$
U_{l} \leq k_{i} q_{s s} \leq U_{h}
$$

There are many cases like startup, speed up, speed down, reversal, and manual/auto control mode changes in the controller operation. Some cases occur in the linear range and some cases occur in both the saturation and the linear range. According to the difference between the initial and the final integral states, three initial conditions can be considered as seen in Fig. 1; (A) higher, (B) equal, and (C) lower. The $P$-axis value of the initial condition is usually dependent upon the speed reference and the $I$-axis value is related to the previous operating conditions. The $P$-axis value of the steady state condition should be zero due to the error integral action and the $I$-axis value is related to the operating conditions such as the load torque and the speed reference. If the PI trajectory is like the one in Fig. 1, the PI control enters into the saturation range rapidly, and the trajectory goes to zero error via an integral state corresponding to the anti-windup scheme. This interval affects only the rise time in the control performance specification and the $\mathrm{P}$ control dominates. The PI trajectory touches the boundary of the linear range if the attractive condition is satisfied [7].

The error dynamics are dependent upon both the selected PI gain and the touch conditions like the integral state value. Fig. 2 shows the error responses according to the touch conditions under the same PI gain condition. It can be seen that the overshoot is related to the relative position of the integral state against the steady state point. When the integral state on the linear range boundary is higher than the steady state, an overshoot is apt to occur in the error response. On the other hand, the error response may be slowed down as the integral state on the linear range boundary is lower than the steady state value. It is noted that the error response can be deteriorated by an improper touch condition even though a proper PI gain is selected.

There are many schemes for finding the proper touch condition, which is called the anti-windup technique. The touch condition is different depending on the anti-windup scheme. The linear range cases are well expressed and controlled by a well-designed PI controller. However, the saturation range cases are not well understood or controlled.

\section{ANTI-WINDUP PI CONTROL SCHEMES}

\section{A. Conditional integration}

Fig. 3 shows an anti-windup PI controller based on the conditional integration method [3]. Integral action is switched on or off depending on the linear range or the saturation range such as:

$$
\dot{q}=\left\{\begin{array}{lll}
e & \text { if } & u=v \\
0 & \text { if } & u \neq v
\end{array} .\right.
$$

The method is also called integrator clamping. There are a few switching conditions to be considered. One simple 


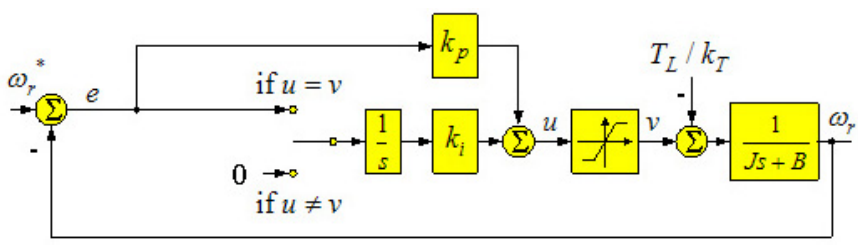

Fig. 3. Conditional integration scheme.

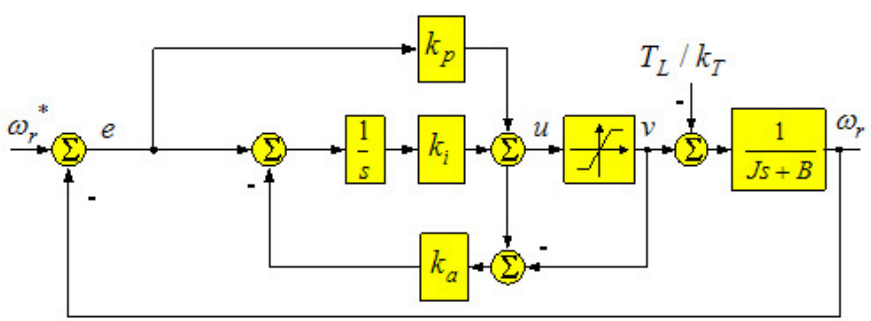

Fig. 4. Tracking back calculation scheme.

approach is to switch off integration during saturation. This method has the disadvantage that the value of the integral part is large when the controller output is different from the plant input. Suppose that the controller is saturated at the upper limit. The integrator is then switched off if the control error is positive, but not if it is negative [1]. Choi et al. [6] have presented a new method based on the conditional integration scheme. The controller changes between the P and PI modes according to specific conditions. However, the main difference is how to set the initial value of the integrator in the PI control mode. The initial value of the integrator is loaded in the $\mathrm{P}$ mode, and the controller works in the PI mode utilizing the loaded initial value. The integrator value is written to compensate for the viscous damping and the load torque at a well-controlled steady state condition without speed error [6]. However, with this method it is difficult to choose a gain to satisfy the anti-windup performance, and the selection of gain is related to the zero of the transfer function. However, the proposed method can result in no overshoot speed response performance.

\section{B. Tracking back calculation}

Fig. 4 shows the tracking back calculation method [6]. In the linear range, the error is integrated and the difference between the saturated and the unsaturated control signals is used to generate a feedback signal to properly control the integral state in the saturation range such as:

$$
\dot{q}=\left\{\begin{array}{ccc}
e & \text { if } \quad u=v \\
e-k_{a}(u-v) & \text { if } \quad u \neq v
\end{array} .\right.
$$

It may seem advantageous to choose a very large value for the anti-windup gain $k_{a}$ because the integrator can be limited quickly. If an anti-windup gain is chosen that is too big, a spurious error can cause input saturation, which accidentally resets the integrator. Usually, the gain $k_{a}$ is selected as $1 / k_{p}$. There is a new scheme for the tracking back calculation method [7]. If the PI controller output is saturated, the integral state is reduced with the rate of the integral time constant by negatively feeding back the controller output. Otherwise, the

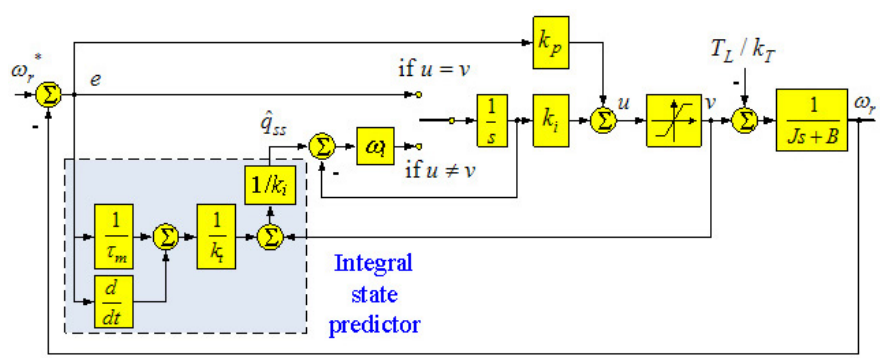

Fig. 5. Integral state prediction scheme.

integral state accumulates a speed error and the PI action is activated.

\section{Integral state prediction}

Fig. 5 shows the integral state prediction (ISP) method [12]. For a step change of the speed reference, the error dynamics can be obtained from (1) and (4) by:

$$
\dot{e}=-\frac{1}{\tau_{m}} e-k_{t} u+T_{l}+\frac{1}{\tau_{m}} \omega_{r}^{*} .
$$

If a PI controlled system is stable, the integral action in the steady state can be expressed as:

$$
k_{i} q_{s s}=\left(T_{l}+\frac{1}{\tau_{m}} \omega_{r}^{*}\right) / k_{t}
$$

where the subscript $s s$ denotes the steady state value. It is noted that the steady state integral action depends on the external load torque and the speed reference. Substituting (10) in to (9) yields the error dynamics as:

$$
\dot{e}=-\frac{1}{\tau_{m}} e-k_{t} u+k_{t} k_{i} q_{s s} .
$$

When PI control operates in the linear range, the output error is connected to the integrator input. When the control operates in the saturation range, the integral state is reset to a predicted steady state value through a low-pass filter to prevent abrupt integral state changing such as:

$$
\dot{q}=\left\{\begin{array}{ccc}
e & \text { if } & u=v \\
\omega_{i}\left(\hat{q}_{s s}-q\right) & \text { if } & u \neq v
\end{array}\right.
$$

where $\hat{q}_{s s}$ is the integral state value in the steady state and it is predicted by using (11) as:

$$
\hat{q}_{s s}=\frac{1}{k_{i}}\left(\frac{1}{k_{t}}\left(\dot{e}+\frac{1}{\tau_{m}} e\right)+v\right) .
$$

The integral state loading time can be determined by adjusting the parameter $\omega_{i}$ properly. Since the integral state prediction in (13) includes the error derivative, the bandwidth of the low-pass filter is constrained by the derivations. In order to achieve the desired performance dynamics specifications while maintaining closed-loop stability, it is therefore important to select an integral state loading time that is faster than the PI controlled system dynamics. 


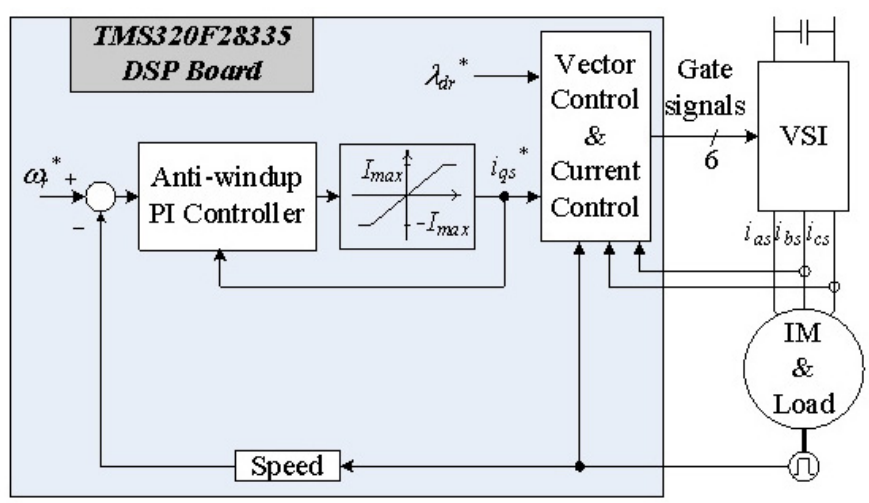

Fig. 6. Block diagram of vector-controlled induction motor drive using anti-windup PI speed controller.

TABLE I

PARAMETERS FOR SIMULATION \& EXPERIMENT

\begin{tabular}{|c|l|}
\hline Motor speed & $1730[\mathrm{rpm}]$ \\
\hline Rated frequency & $60[\mathrm{~Hz}]$ \\
\hline Saturation limited range & {$[-7,7][\mathrm{A}]$} \\
\hline Torque constant $\left(K_{T}\right)$ & $1.3[\mathrm{Nm} / \mathrm{A}]$ \\
\hline Moment of inertia $(J)$ & $5.710^{-3}[\mathrm{Kgm} 2]$ \\
\hline Viscous damping coefficient $(B)$ & $1.65 \times 10^{-3}[\mathrm{Kgm} 2 / \mathrm{s}]$ \\
\hline
\end{tabular}

\section{EXPERIMENTAL COMPARISON OF} ANTI-WINDUP PI CONTROL SCHEMES

In order to compare and evaluate anti-windup PI control schemes, the speed control of an induction motor has been implemented and experimented on. The parameters of a 1-hp induction motor are listed in Table I. Fig. 6 shows a block diagram of the experimental system. In the vector control method, the induction motor is controlled like a separately excited dc motor. The control algorithm is fully implemented in software with a TMS320F28335 DSP, which includes A/D converters and a three-phase PWM generator. The three-phase currents are controlled to settle within $2 \mathrm{~ms}$ by using a synchronous PI regulator and the rotor flux is controlled to settle within $40 \mathrm{~ms}$. The space vector PWM method is used and the PWM frequency is $10 \mathrm{KHz}$. The sampling times of the current and the speed control loops are $0.1 \mathrm{~ms}$ and $2 \mathrm{~ms}$, respectively. The shaft encoder has 1,000 pulses per revolution. Among the various anti-windup PI controllers, the conditional integration, the tracking back calculation, and the proposed ISP schemes are compared.

The PI gains are chosen as a rise time of $0.1 \mathrm{sec}$ and a damping ratio of 1 . The gain $k_{a}$ in the tracking back calculation is taken as $1 / k_{p}$. The parameter $\omega_{i}$ is selected as a $1 /(0.5 *$ rise time).

Fig. 7 shows the experimental results of the anti-windup schemes at no load. The base values of the speed and current are $1800 \mathrm{rpm}$ and $2.77 \mathrm{~A}$, respectively. It can be seen from Fig. 7(c) that the magnitude order of the integral state value, compared with its steady state value, is the conditional integration, the ISP, and the tracking back calculation when the PI controller enters the linear range. Therefore, the tracking back calculation method has the largest overshoot in speed response and the proposed ISP scheme has a similar response to the conditional integration scheme as shown in Fig 7(a).

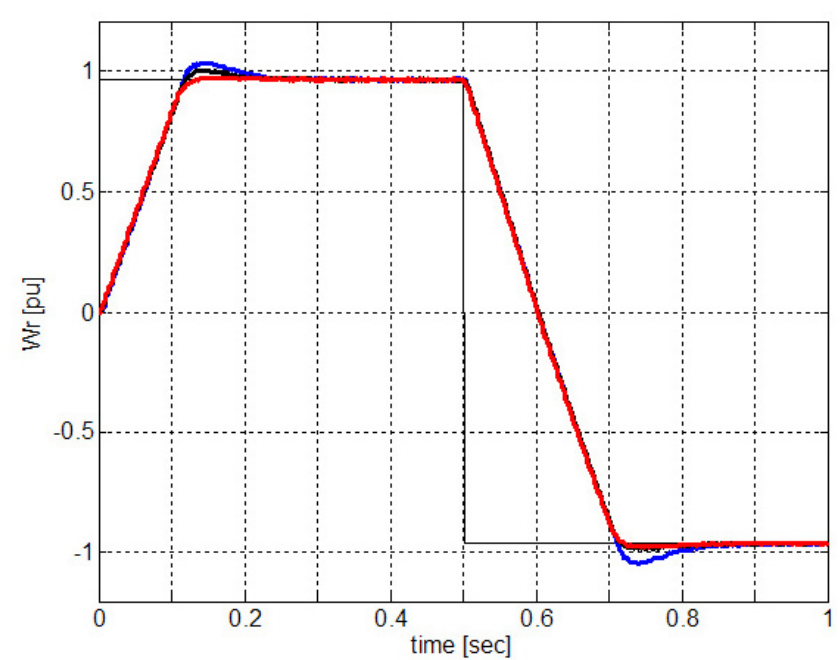

(a)

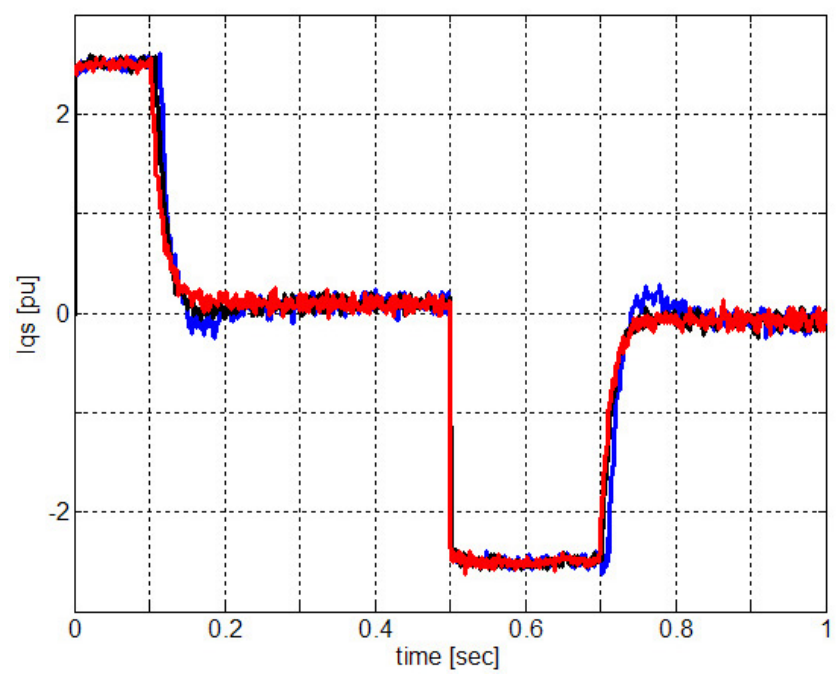

(b)

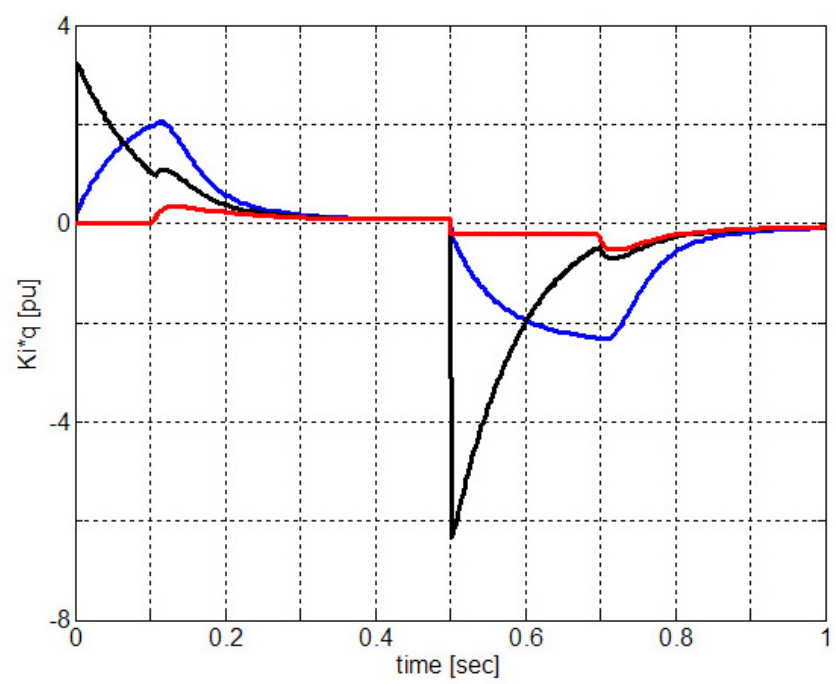

(c)

Fig. 7. Experimental comparison at no load. (a) speed response. (b) torque producing currents. (c) integral states. (dotted: conditional integration, dashed: tracking back calculation, solid: proposed ISP). 


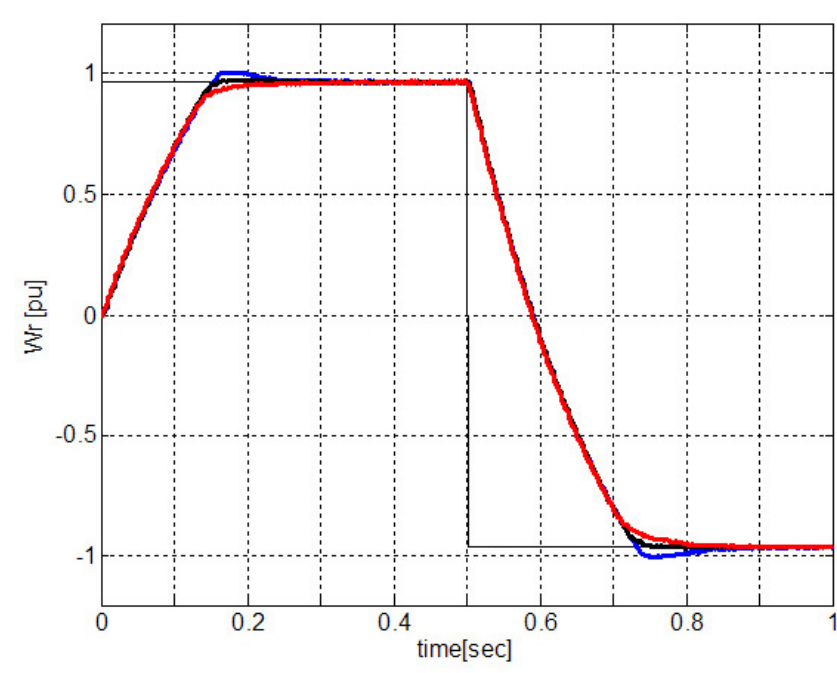

(a)

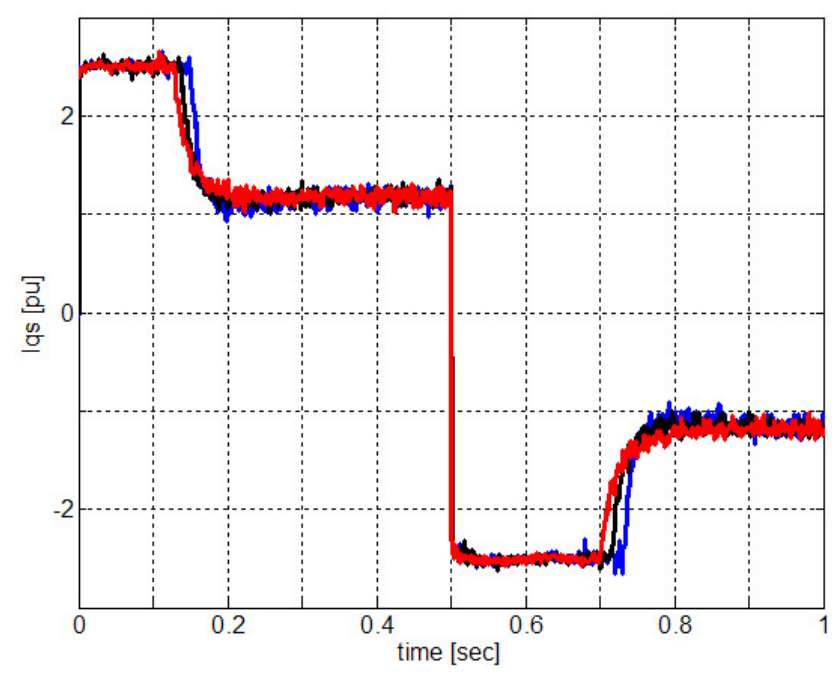

(b)

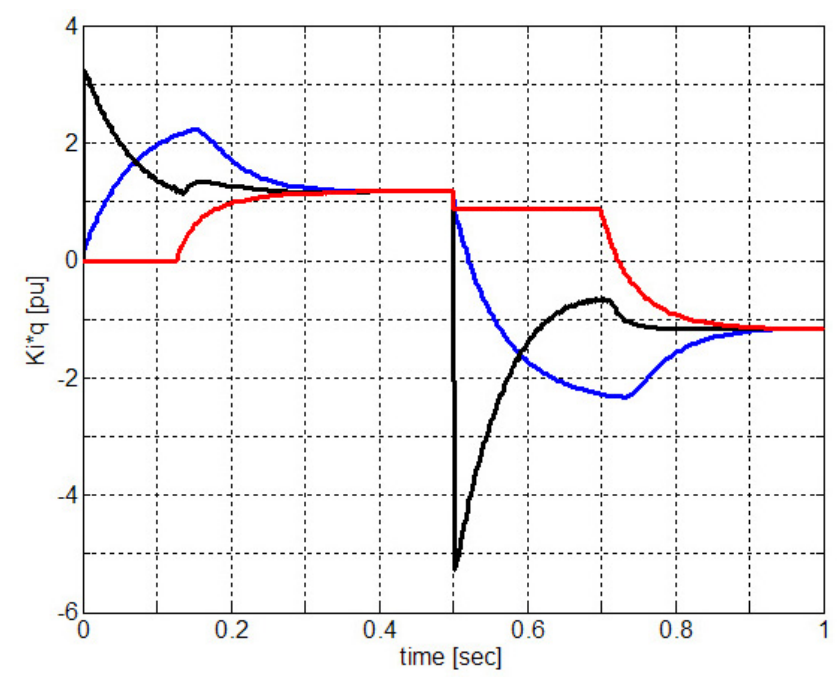

(c)

Fig. 8. Experimental comparison at full load. (a) speed response. (b) torque producing currents. (c) integral states. (dotted: conditional integration, dashed: tracking back calculation, solid: proposed ISP).
Compared with the PI controlled system, the integral state loading of the ISP has slow dynamics because the speed sensing from the encoder has a high noise at the low speed range and the predicted integral state also has noise due to the differential action. Therefore, it can be seen from Fig. 7(c) that the integral state of the ISP cannot reach its steady state value at the startup condition but that it reaches near the steady state value at the speed reversal condition.

Fig. 8 compares experimentally the speed responses of the anti-windup controllers at full load. When the PI controller enters into the linear range, the integral state of the ISP scheme goes into its steady state value as seen in Fig. 8(c). Hence, the speed output has nearly the same responses in both the startup and the reversal conditions. In the case of the conditional integration, the integral state value is much lower than its steady state value at the beginning of the linear range, so that the speed response is overdamped as shown in Fig. 8(a). For the tracking back calculation method, the integral state has a larger magnitude than the steady state and an overshoot occurs in the speed response.

From the PI plane analysis and the experimental results, the transient response of the anti-windup PI controllers can be considered as a saturation range response and a linear range response. The saturation range response is related to the rise time of the PI system and it depends mainly on the control output limit value and the $\mathrm{P}$ gain. The linear range response is related to the control performances such as the overshoot and the settling time. The linear performance depends on both the PI gain and the initial integral state at the beginning of the linear range. The PI gain determines the system stability and the initial integral state value is very important for obtaining a well-designed transient response.

\section{CONCLUSIONS}

An anti-windup PI control scheme for variable-speed motor drives has been proposed in order to overcome the windup phenomenon and some representative anti-windup PI controllers have been compared and evaluated. The control schemes have been applied to the speed control of a vector-controlled induction motor driven by a PWM-VSI and its usefulness has been experimentally verified.

The PI plane is very useful for evaluating the anti-windup PI controller. The rise time is mainly related to the control input limit during the saturation range regardless of the anti-windup scheme. Also, the control performances such as the overshoot and the setting time depend on the touching condition in the PI plane rather than the PI gains.

\section{REFERENCES}

[1] K. Astrom, and T. Hagglund, PID Controllers: Theory, Design, and Tuning, Research Triangle Park, NC: ISA, Jan. 1995.

[2] N. J. Krikelis and S. K. Barkas, "Design of tracking systems subject to actuator saturation and integral wind-up," Int. J. Control, Vol. 39, No.4, pp. 667-682, Jul. 1984.

[3] R. Hanus, M. Kinnaert, and J. L. Henrotte, "Conditioning technique, a general anti-windup and bumpless transfer method," Automatica, Vol. 23, No. 6, pp. 729-739, Nov. 1987. 
[4] J. K. Seok, K. T. Kim, and D. C. Lee, "Automatic mode switching of P/PI speed control for industry servo drives using online spectrum analysis of torque command," IEEE Trans. Ind. Electron., Vol. 54, No. 5, pp. 2642-2647, Oct. 2007.

[5] K. S. Walgama, S. Ronnback, and J. Sternby, "Generalization of conditioning technique for anti-windup compensators," Proc. Inst. Elect.Eng., Vol. 139, pt. D, No. 2, pp. 109-118, Mar. 1992.

[6] J. W. Choi and S. C. Lee, "Antiwindup strategy for PI-type speed controller," IEEE Trans. Ind. Electron., Vol. 56, No. 6, pp. 2039-2046, Jun. 2009.

[7] H. B. Shin, "New antiwindup PI controller for variable-speed motor drives," IEEE Trans. Ind. Electron., Vol. 45, No. 3, pp. 445-450, Jun. 1998.

[8] J. G. Park, J. H. Chung, and H. B. Shin, "Anti-windup integralproportional controller for variable-speed motor drives," J. of Power Electronics, Vol. 2, No. 2, pp. 130-138, Apr. 2002.

[9] K. Ohishi, E. Hayasaka, T. Nagano, M. Harakawa, and T. Kanmachi, "High-performance speed servo system considering voltage saturation of a vector-controlled induction motor," IEEE Trans. Ind. Electron., Vol. 53, No. 3, pp. 795-802, Jun. 2006.

[10] N. J. Krikelis, "State feedback integral control with intelligent integrator," Int. J. Control, Vol. 32, No. 3, pp. 465-473, May 1980.

[11] J. G. Park, J. H. Chung, and H. B. Shin, "Anti-windup integralproportional controller for variable-speed motor drives," J. of Power Electronics, Vol. 2, No. 2, pp. 130-138, Apr. 2002.

[12] H. B. Shin, S. Lee, J. M. Park, and H. S. Shin, "Anti-windup PID controller," Korea Patent, 2010-0014689, Feb. 18, 2010.

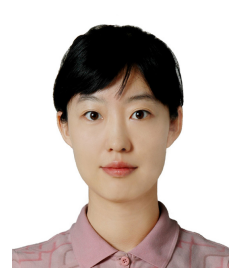

Xin-lan Li received her B.S. in Automatic Control from the University of Science and Technology Liaoning, Liaoning, China, in 2004, and her M.S. in Electrical Engineering in 2006 from Gyeongsang National University, Gyeongnam, Korea, where she is currently working towards her Ph.D. in the Department of Electrical Engineering and Electronics. Her current research interests include the control of power systems and power electronics.

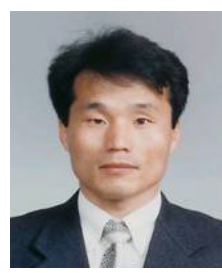

Jong-Gyu Park received his B.S., M.S., and Ph.D. in Electrical Engineering from Gyeongsang National University, Korea, in 1995, 1997, and 2004, respectively. He is currently a Visiting Associate Professor at Namhae College, Gyeongnam, Korea. His research interests include power electronics, ac machine drives, and microcontroller-based control applications.

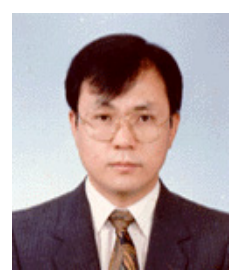

Hwi-Beom Shin (SM'86, M'95) received his B.S. in Electrical Engineering from Seoul National University, Seoul, Korea, in 1982, and his M.S. and Ph.D. also in Electrical Engineering from the Korea Advanced Institute of Science and Technology (KAIST), Seoul, Korea, in 1985 and 1992, respectively. He was with Hyundai Electronics Industries Co. Ltd. as Chief Engineer from 1990 to 1992. Since 1993, he has been with the Department of Electrical Engineering, Gyeongsang National University, Gyeongnam, Korea, where he is a Professor and a Researcher in the Engineering Research Institute. His research interests are in the areas of power electronics and control, electric vehicles and industrial drives. Prof. Shin is a member of the Korea Institute of Power Electronics, the Korea Institute of Electrical Engineering, and the IEEE Power Electronics and Industrial Electronics Societies. 